\title{
UN NUEVO ENFOQUE EN LA ENSEÑANZA DE LA QUIMICA*
}

\section{Identificación del problema:}

La Química parece "Ciencia acabada”. Actualmente, en la enseñanza de la Química, existe la separación entre la docencia y la investigación. El profesor hace una explicación lo más acabada posible sobre los fenómenos, la cual se convierte para los estudiantes en la única interpretación de ellos.

El universo se comporta como se afirma en clase; sí un fenómeno se escapa a esta forma de ver la realidad, es que no cumple la ley, y no que ésta sea inacabada, incompleta.

La enseñanza es ideológica, no científica: Para estudiantes y profesores no se trata de estudiar en términos analógicos y críticos la estructura conceptual de la Química, sino de recibir y dar información cargada de opiniones: respuestas a aprender.

La Química vista así, no es una ciencia con modelos probables de su objeto de conocimiento que tienen en el fondo interrogantes inherentes a su mismo proceso de producción; que hay que investigar, sino estructuras rígidas; afirmaciones que hay que memorizar. El mejor estudiante no es quien se dedica a la búsqueda de elementos y conexiones que redefinan los modelos conceptuales, sino el que repita en forma más completa las definiciones dadas por el profesor a los textos.

Lo anterior es una de las causas por las cuales los estudiantes no manifiestan un deseo de investigar, ni tampoco adquieren la formación del espíritu científico que caracteriza a quienes se dedican al conocer.

El objetivo de cada curso es meramente informativo: Se eliminan todas las otras etapas que definen el proceso de producción de la estructura conceptual de una ciencia y por lo tanto no se hace Química sino que se llena a los estudiantes de respuestas de Química.

El profesor se rutiniza: Tiene como objetivo informar, por eso sólo aprende el conjunto de informaciones que constituyen su curso, según el programa diseñado, para dárselas a sus estudiantes. Una vez que el aprendizaje del profesor se ha dado, su problema como docente finaliza y repite todos los años lo mismo.

\section{Objetivos:}

La propuesta de este nuevo enfoque (Q - U.P.N.) pretende mejorar la enseñanza de la Química a todos los niveles del sistema educativo, además de:

1. Eliminar la dualidad Docencia-Investigación a través de:

\footnotetext{
* Esta ponencia será presentada por profesores de Química de la Universidad Pedagógica Nacional, en el Seminario Nacional sobre Química a realizarse próximamente en el pals.
} 
a) El diseño de cada curso como un proceso de conocimiento emprendido por el profesor y sus alumnos.

b) La toma de cada elemento de aprendizaje como un problema de investigación.

2. Formar en los estudiantes un espíritu científico.

3. Despertar en los estudiantes el interés por la investigación científica.

4. Transformar el "dictar" clase por el "Hacer" Química.

5. Eliminar la rutinización del profesor.

\section{Marco teórico:}

Es común decir al comienzo de los cursos de Química que ésta es una ciencia, como un formulismo meramente, que luego en el desarrollo de las clases se olvida o no se tiene en cuenta.

La Química es una ciencia porque está constituida por un cuerpo de conceptos organizados estructuralmente, con reglas de producción que constituyen la directriz básica para estudiar, descubrir y analizar los problemas que se plantean a su interior. La Química es fruto del quehacer científico del hombre en el campo de la composición, estructura y comportamiento de las sustancias, este quehacer es un proceso de conocimiento de su objeto, que conduce a formular modelos de él, que son tanto más completos cuando mayor reflejen la realidad, pero ésta es compleja, por lo tanto cada modelo está planteado en términos de potencias, de probabilidad. El objetivo de la investigación es "ver" hasta qué punto el modelo se comporta conforme al objeto para el cual se ha elaborado, (No es que la realidad se comporte conforme el modelo). Surge entonces la experimentación como una necesidad del proceso.

Cuando el hombre estudia, lo hace en términos de enfrentamiento con su objeto de conocimiento. Este enfrentamiento es una interacción de su cuerpo de conciencia con el mundo exterior en términos de interrogantes, formulaciones que llevan a conceptualizaciones, (las hipótesis), que originan procedimientos para revisar y someter a prueba toda la estructura elaborada; de aquí surgirán nuevas formulaciones que conducirán necesariamente hacia abstracciones cada vez más complejas. Cuando la complejidad sea tan grande como la realidad, entonces nuestro modelo de ella será el de mayor probabilidad.

Si como fruto de nuestro trabajo elaboramos respuestas y no interrogantes caemos en el campo de la ideología, que es el no saber científico. Cuando se tienen respuestas para todo, no se sabe nada; se sabe algo cuando no se tienen.

\section{El nuevo enfoque:}

La Química no es una ciencia acabada, los modelos que posee de su objeto tienen imperfecciones, poseen multitud de interrogantes que hacen que valga la pena embarcarse en la aventura de su elucidación.

Decidirse por estudiar ciencias, cualquiera que ésta sea, se debe a que tenemos conciencia de un "no" saber y una gran capacidad de maravillarnos ante la complejidad de 
la realidad. Nos interrogamos acerca de ella, analizamos los modelos que se han dado y encontramos fallas, imprecisiones que nos mueven a interesarnos por la investigación.

Enseñar debe ser, por lo tanto, orientar al estudiante para que emprenda esta actividad, pero el orientador debe ser también un aprendiz, con la diferencia entre él y sus estudiantes en el hecho de que se embarcó antes que ellos en la aventura del conocimiento y por lo tanto los interrogantes que formula acerca del objeto de conocimiento son más profundos, tienen un mayor nivel de complejidad. En conjunto trabajan en la búsqueda de nuevas implicaciones en la estructura conceptual de la ciencia que les interesó. Aprenden haciendo. Cada semestre o año el profesor planea sus cursos como un trabajo de investigación, bajo las nuevas perspectivas que en el período anterior obtuvo, replantea los problemas de la realidad bajo las nuevas formulaciones que surgieron acerca de ella cuando trabajó con sus estudiantes en el curso pasado. La labor educativa se transforma de esta manera en una actividad dinámica donde los estudiantes no se aburren ni el profesor se rutiniza, y cada curso es una oportunidad para avanzar.

Ahora surge una pregunta: ¿Cómo evaluar a sus estudiantes y el curso? Los exámenes se harán con el objeto no de obtener respuestas, sino que dado un problema deben vislumbrar el mayor número de interrogantes que sirvan de directriz para el estudio. Se evalúa no que saben los estudiantes, sino la profundidad de las conexiones que ven al rededor de un objeto de conocimiento, fruto del análisis lógico y científico de su fenomenología. De acuerdo con la profundidad del análisis el profesor evalúa el procedimiento diseñado para realizar el trabajo de aprendizaje. Cuando surgen dificultades de aprendizaje, profesor y alumnos descubren la forma de superarlos, dado que al estar trabajando sus problemas, realizan su camino y pueden ver qué los detiene, qué les impide seguir adelante, pueden analizar los factores que crean las dificultades y producir las soluciones adecuadas.

\section{Medios para lograr los objetivos:}

Para lograr convertir la clase en una actividad de conocimiento, el profesor debe planear su trabajo en el sentido de una investigación, lo cual se puede conseguir con guías para que los estudiantes planteen y formulen interrogantes sobre un hecho. Lo ideal sería que el profesor organizara las guías junto con sus estudiantes de acuerdo con sus intereses, para que den las direcciones de investigación y el tipo de enfrentamiento con el problema que consideren adecuado.

El profesor dividirá operativamente su labor así:

\section{Identificación del problema de conocimiento}

Este problema podría ser una unidad dentro del programa analítico del curso que contendría una serie de subunidades o subtemas y las actividades serian:

a) Formulación y análisis de todos los interrogantes que surgen al rededor del problema a aprender.

b) Formulación de métodos que llevarían a la producción de una estructura conceptual acerca de él.

\section{Investigación bibliográfica}


Búsqueda de información sobre los modelos existentes acerca del objeto de conocimiento.

a) Análisis crítico.

b) Formulación de interrogantes e identificación de fallas.

3. Diseño y ejecución de un proceso experimental.

4. Confrontación de la información bibliográfica y experimental.

A la luz de este análisis debe surgir la reformulación del modelo actual e instancias investigativas. Esta etapa debe dar el marco teórico que lleve a la necesidad de una profundización de ir más allá.

\section{Dificultades que surgen en la aplicación del nuevo enfoque:}

\section{Ejecución de un programa de curso}

El hecho de cumplir con un programa hace que no se pueda detener tanto cuanto se quiera en una unidad, dado que hay que pasar a la siguiente a fin de que los estudiantes tengan los elementos suficientes para manejar otros elementos teóricos en el próximo curso.

En el Departamento de Química de la Universidad Pedagógica Nacional, estamos actualmente obviando esta dificultad con el método Q-UPN aplicado a nivel del bachillerato (IPN), que consiste en:

1.1 División del curso en Unidades y subunidades y elaboración de guías de trabajo, para cada subtema.

Estas guías son de dos tipos: (Integradas).

Teóricas y de laboratorio

Cada guía teórica consta de:

a) Nombre de la guía.

b) Número.

c) Objetivos generales de la unidad.

d) Objetivos específicos del subtema.

e) Preguntas acerca del subtema.

f) Información bibliográfica.

g) Cuestionario de evaluación. 
Cada guía de laboratorio consta de:

a) Título.

b) Fundamentos teóricos (el modelo conceptual que se pretende atacar).

c) Materiales y activos.

d) Procedimiento.

e) Tabla de datos.

f) Cuestionario de evaluación.

1.2 Formación de grupos de cuatro estudiantes máximos para el trabajo, supervisados por el profesor.

1.3 El tiempo de aula-clase se ha diseñado dentro de un tiempo total de dos horasclase, así:

a) 60 minutos durante los cuales los grupos estudian las preguntas formuladas en la guía, con auxilio de la bibliografía y del profesor.

b) 30 minutos de discusión, durante los cuales el profesor busca confrontaciones conceptuales entre los grupos, e interrogantes acerca del problema.

1.4 El profesor orienta la clase de tal forma que los interrogantes que surjan, desemboquen en el problema que se plantea en la guía siguiente.

1.5 Los otros interrogantes que surgen constituyen las tareas o trabajos fuera de aula.

1.6 Con las guías de laboratorio ocurre otro tanto, se analizan y discuten los resultados, con el objeto de atacar el modelo conceptual que le sirve de fundamento teórico y que se integran al análisis y discusión del trabajo de aula-clase.

\section{Recursos bibliográficos}

La escasez de recursos económicos de los estudiantes para adquirir textos y revistas y la pobreza de las bibliotecas.

\section{Laboratorio}

Este problema se supera diseñando experiencias no sofisticadas utilizando los recursos del medio y en esto el profesor tanto como sus estudiantes requieren de ingenio e inventiva.

\section{La falta de conocimientos previos}

Este es un mito que han levantado los empiristas alrededor del método científico, lo cual quiere decir que el hombre requirió inicialmente de un Ángel o cualquiera deidad que le suministrara la información previa para comenzar a atender el mundo. Conocer es una actividad en el presente continuo, que le permite al hombre formular modelos sobre la 
realidad. En la medida que se va dando el proceso surgen las dificultades, entre ellas, la necesidad de estudiar algunos fenómenos correlativos que permitan una mayor formulación de nuestro problema, es entonces cuando se buscan las formas de allegar los elementos teórico-prácticos requeridos.

5. A nivel universitario hemos diseñado la siguiente estrategia (Q-U.P.N. -2).

5.1 El contenido del curso se divide en problemas de conocimiento, constituye cada problema una unidad.

5.2 División del curso en grupos de tres estudiantes.

5.3 El problema propuesto es atacado en cada grupo independiente.

5.4 Uno de los grupos conduce conjuntamente con el profesor el desarrollo del análisis del problema para lo cual, debe:

a) Diseñar guías teórico-prácticas.

b) Dirigir el análisis de práctica.

c) Aumentar la discusión del marco teórico del problema.

d) Establecer, con el grupo total de estudiantes, fallas e inconsistencia de cada modelo y proponer procesos teóricos y experimentales para superarlos.

6. La evaluación se hace:

a) Personalmente a través del trabajo, en la medida que los estudiantes superan obstáculos.

b) Mediante la confrontación entre grupos de argumentos consistentes acerca del problema.

c) De la estrategia diseñada en la producción de conocimientos, mediante una prueba final en la cual los estudiantes formularán interrogantes acerca de un problema.

El profesor rediseñará la estrategia seguida de acuerdo a los resultados obtenidos en el curso para mejorar el siguiente. 